BULLETIN OF THE

AMERICAN MATHEMATICAL SOCIETY

Volume 79, Number 3, May 1973

\title{
LINK MANIFOLDS AND PERIODICITY
}

\author{
BY LOUIS H. KAUFFMAN ${ }^{1}$
}

Communicated by William Browder, November 6, 1972

1. Introduction. This research was motivated by a number of considerations not the least of which was an interesting phenomenon of periodicity observed by Alan Durfee [1]. Letting $M_{k}$ denote the Brieskorn variety $V\left(Z_{0}^{k}+Z_{1}^{2}+Z_{2}^{2}+\cdots+Z_{n}^{2}\right) \cap S^{2 n+1}$ for $n \geqq 3$, odd and $k=1,2,3, \ldots$, he finds that $M_{k} \simeq M_{k+8}$ where $\simeq$ denotes diffeomorphism. It was pleasant to speculate on possible geometric explanations for this regularity.

We examine this in the context of transformation groups. $M_{k}$ is an $O(n-1)$-manifold with three orbit types and orbit space $D^{4}$. The fixed point set corresponds to a torus link in $S^{3}=\partial D^{4}$. Extending results of Hirzebruch and Erle [2] to the case of links we obtain a classification for these "link manifolds" in terms of invariants of links. These results are stated in $\S \S 2$ and 3. $\S 4$ discusses periodicity. Proofs will appear elsewhere.

2. Invariants of links. Given a link $L \subset S^{3}$ together with assigned orientations for its components, one may form an oriented surface $F \subset S^{3}$ such that $\partial F=L$ and $F$ induces the given orientation on $L$. The Seifert pairing is a bilinear map $\theta: H_{1}(F) \times H_{1}(F) \rightarrow Z$ given by $\theta(x, y)=l\left(i_{*} x, y\right)$ where $i_{*}$ denotes "the push off of $F$ in the direction of the positive normal" and $l($,$) is the linking number in S^{3}$. One then defines an even, symmetric bilinear form $f: H_{1}(F) \times H_{1}(F) \rightarrow Z$ by $f(x, y)=\theta(x, y)+\theta(y, x)$. It turns out that the signature of $f$ is an invariant of link type. In fact, it is identical with the Murasugi signature of the link [3]. We denote it by $\sigma(L)$. Let $A f: H_{1}(F) \rightarrow \operatorname{Hom}\left(H_{1}(F), Z\right)$ be the adjoint map $A f(x)(y)=f(x, y)$ and let $G(L)=$ cokernel $(A f)$. Then $G(L)$ is also an invariant of $L$; it is the first homology group of the double branched cover of $S^{3}$ with branch set $L$.

Let $\tau G(L)$ be the torsion subgroup. Then there is a quadratic form $q(L): \tau G(L) \rightarrow Q / Z$. The equivalence class of this form is an invariant of $L$; it may be identified with the linking form of the double branched cover.

\section{3. $O(n)$-manifolds.}

DEFINITION. A link-manifold $M^{2 n+1}$ is a smooth closed manifold admitting a smooth action of the orthogonal group $O(n)$ such that all isotropy subgroups are conjugate to $O(n), O(n-1)$ or $O(n-2)$, the orbit space is diffeomorphic to $D^{4}=\left\{x \in R^{4} \mid\|x\| \leqq 1\right\}$ and the fixed point set in $M$ corresponds to a link $L \subset S^{3}=\partial D^{4}$.

AMS (MOS) subject classifications (1970). Primary 57E25.

${ }^{1}$ Supported in part by NSF grant no. GP-28487. 
Let $B_{2 n+2}$ denote the set of diffeomorphism classes of $O(n)$ link manifolds.

Definition. $B P_{2 n+2} \equiv$ the set of diffeomorphism classes of $(n-1)$ connected $(2 n+1)$-manifolds which bound parallelizable manifolds.

THEOREM 1. (a) $B_{4 k} \equiv B P_{4 k}$ for $k>1$.

(b) Suppose $M_{1}, M_{2} \in B_{4 k}, k>1$, and $M_{i} \leftrightarrow L_{i} \subset S^{3}$.

$$
G\left(L_{1}\right) \simeq G\left(L_{2}\right), \quad q\left(L_{1}\right) \simeq q\left(L_{2}\right), \quad \sigma\left(L_{1}\right) \geqq \sigma\left(L_{2}\right) .
$$

Then

$$
M_{1} \simeq M_{2} \# \frac{1}{8}\left(\sigma\left(L_{1}\right)-\sigma\left(L_{2}\right)\right) \cdot \Sigma
$$

where \# denotes connected sum and $\Sigma$ is the Milnor sphere.

REMARK. A link manifold automatically determines an orientation for the corresponding link so that the above invariants are defined.

It is not true that $B_{2(2 k+1)}=B P_{2(2 k+1)}$. However $B_{2(2 k+1)} \subset B P_{2(2 k+1)}$ and these manifolds may also be classified, this time in terms of $Z_{2}$ quadratic forms for the links. We omit discussion of these quadratic forms and only state

THEOREM 2. Let $n$ be odd, $n \geqq 3, T=$ tangent sphere bundle to $S^{n}$, $\Sigma_{1}=$ Kervaire Sphere of dimension $2 n-1$. Then $B_{2 n}$ consists precisely of the manifolds

$$
\begin{gathered}
\left(S^{n-1} \times S^{n}\right) \#\left(S^{n-1} \times S^{n}\right) \# \cdots \#\left(S^{n-1} \times S^{n}\right), \\
T \# T \# \cdots \# T, \\
\left(S^{n-1} \times S^{n}\right) \# \cdots \#\left(S^{n-1} \times S^{n}\right) \# \Sigma_{1}, \\
S^{2 n-1}, \Sigma_{1} .
\end{gathered}
$$

Manifolds corresponding to knots are just $\Sigma_{1}$ or $S^{2 n-1}$. If a link has $r+1$ components then $T$ or $S^{n-1} \times S^{n}$ will appear $r$ times in the connected sum. Mod 2 quadratic forms for the link detect the appearance of $S^{n-1} \times S^{n}, T$ and $\Sigma_{1}$.

4. Periodicity. Given a link $L \subset S^{3}$ and a connected oriented surface $F \subset S^{3}$ with $\partial F=L$, the surface $F$ may be represented as a disk with attached bands. Given two bands on the surface one may imagine passing one band through the other replacing an over-crossing by an undercrossing (see Figure 1). The same operation may be performed on a single band as in Figure 2. The result is a new surface and a new link. We say that $L$ is pass-equivalent to $L^{\prime}\left(L \sim L^{\prime}\right)$ if $L^{\prime}$ is obtained from $L$ by a sequence of such band-passes.

Fact. If $M_{1}, M_{2} \in B_{2 n}$ for $n$ odd $\geqq 3$ with $M_{i} \leftrightarrow L_{i} \subset S^{3}$ and $L_{1} \sim L_{2}$, then $M_{1} \simeq M_{2}$. 

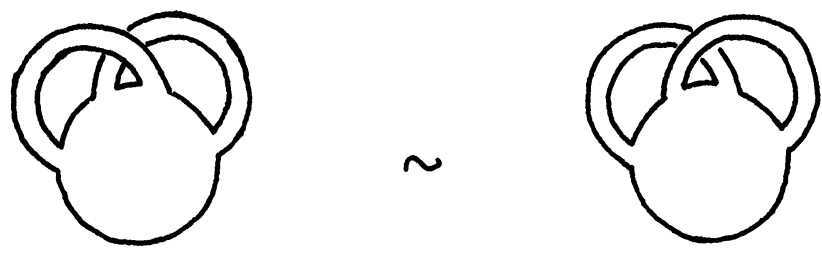

FIGURE 1
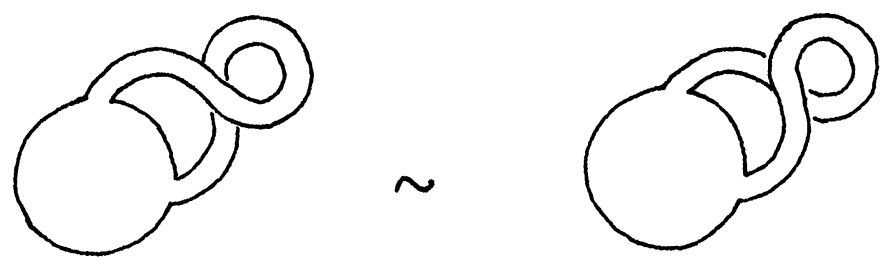

FIGURE 2

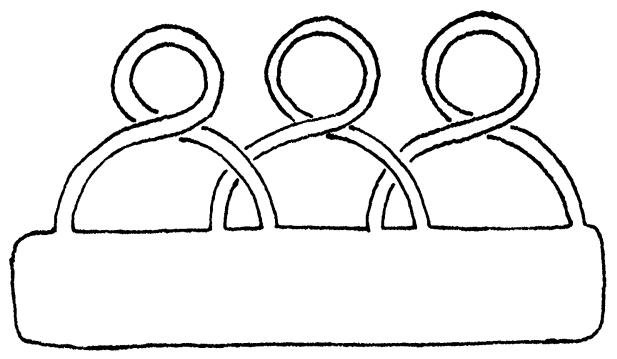

FIGURE 3

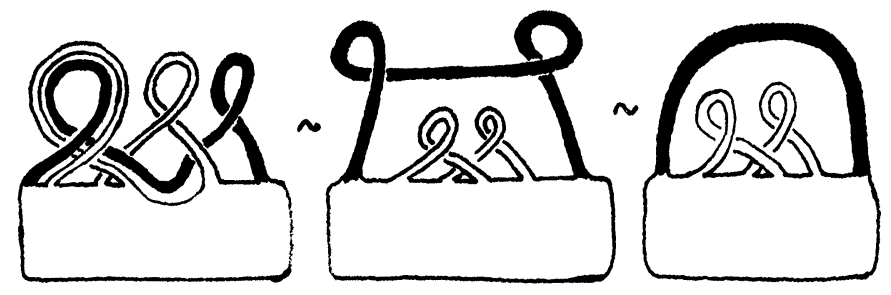

Figure 4 
ReMarK. This follows from [2]. $M_{1}$ and $M_{2}$ may be obtained by explicit surgery on a sphere corresponding to the band representations for the spanning surfaces of the links. Band-passing amounts to certain isotopies of the handles.

Our strategy for exhibiting the periodicity is to begin with a $(2, k)$-torus link having appropriate spanning surface and then simplify it by passequivalence. Figure 3 indicates a $(2,4)$-link with spanning surface. In Figure 4 we show an embedded surface isotopic to that of Figure 3 and a sequence of pass-equivalences. These show that the $(2,4)$-link is $\sim$ a $(2,3)$-link connected sum with a trivial link of two circles. This then implies that $M_{4} \simeq M_{3} \# S^{n-1} \times S^{n}$. Similarly, one finds that $M_{1} \simeq S^{2 n-1}$, $M_{2} \simeq T, M_{3} \simeq \Sigma_{1}$ and

$$
\begin{aligned}
M_{2 k+2} & \simeq M_{2 k+1} \# S^{n-1} \times S^{n}, & & k \text { odd }, \\
& \simeq M_{2 k+1} \# T, & & k \text { even, } \\
M_{2 k+3} & \simeq M_{2 k+1}, & & k \text { odd }, \\
& \simeq M_{2 k+1} \# \Sigma_{1}, & & k \text { even } .
\end{aligned}
$$

Thus

$$
\begin{array}{ll}
M_{1} \simeq S^{2 n-1}, & M_{2} \simeq T, \\
M_{3} \simeq \Sigma_{1}, & M_{4} \simeq \Sigma_{1} \# S^{n-1} \times S^{n}, \\
M_{5} \simeq \Sigma_{1}, & M_{6} \simeq \Sigma_{1} \# T \simeq T, \\
M_{7} \simeq \Sigma_{1} \# \Sigma_{1} \simeq S^{2 n-1}, & M_{8} \simeq S^{n-1} \times S^{n},
\end{array}
$$

and since $M_{9} \simeq S^{2 n-1}$ the list begins repeating and we conclude that $M_{k+8} \simeq M_{k}$.

REMARK. The diffeomorphisms $\Sigma_{1} \# T \simeq T$ and $M_{3} \# M_{3} \simeq S^{2 n-1}$ may also be proved by band-passing. Since $M \# M \simeq S^{2 n-1}$ implies that $M$ is homeomorphic to a sphere, this constitutes an "elementary" proof that the Brieskorn variety $M_{3}$ is homeomorphic to a sphere.

\section{REFERENCES}

1. A. Durfee, Diffeomorphism classification of isolated hypersurface singularities, Thesis, Cornell University, Ithaca, N.Y., 1971.

2. D. Erle, Die quadratische Form eines Knotens und ein Satz über Knotenmannigfaltigkeiten, J. Reine Angew. Math. 236 (1969), 174-217. MR 40 \#2106.

3. K. Murasugi, On a certain numerical invariant of link types, Trans. Amer. Math. Soc. 117 (1965), 387-422. MR 30 \# 1506.

Department of Mathematics, University of Illinois at Chicago Circle, Chicago, ILLINOIS 60680 